\title{
Validation of an empirical method for thin cirrus correction with Sentinel-2 data
}

\author{
S. Salgado ${ }^{\mathrm{a}, \mathrm{b}}$, L. Poutier ${ }^{\mathrm{b}}$, X. Briottet ${ }^{\mathrm{b}}$, S. Mathieu ${ }^{\mathrm{c}}$ \\ aniversité Fédérale Toulouse Midi-Pyrénées, 41 Allée Jule Guesde, 31013 Toulouse Cedex 6, \\ France; \\ bONERA-The French Aerospace Lab, 2 Avenue Edouard Belin, 31055 Toulouse Cedex 4, France ; \\ ${ }^{\mathrm{C}}$ Thales Alenia Space, 5 Allée des Gabians, 06156 Cannes la Bocca Cedex France
}

\begin{abstract}
Most satellite images are polluted by the presence of semi-transparent clouds, called cirrus, returning them unusable. Gao and $\mathrm{Li}$ (2017) have developed an empirical method for the correction of thin cirrus for Landsat-8, allowing to return to a clear sky apparent reflectance. Validated qualitatively, we propose here a quantitative validation method using Sentinel-2 data by comparing the corrected image with a clear sky reference image. Their method shows good results on dark surfaces, like water, with an apparent reflectance found close to 0.02 . On the other hand, it becomes less accurate for thicker cirrus and on more reflective surfaces. In addition, the data analysis shows that pixels located in the shadow of the cirrus are over-corrected. The downward path should therefore be taken into account when correcting the signal.
\end{abstract}

Keywords: Cirrus, cloud, correction, validation, atmosphere, cirrus removal

\section{INTRODUCTION}

Atmospheric correction is today well controlled under clear-sky conditions. Unfortunately, an average of 2/3 of the Earth are covered by clouds and at the scale of an observed scene of a few tens or even hundreds of $\mathrm{km}^{2}$, they can cover the sky entirely, reducing drastically the amount of usable data acquired by an optical sensor from space. Among the different types of clouds, the most widespread are cirrus clouds, with an average coverage of around $30 \%{ }^{1}$

Cirrus are semi-transparent clouds, allowing us to see the underlying surface. Thus, there is a real interest to achieve an atmospheric correction in the presence of such clouds if their optical thickness is not too thick. These clouds, composed with ice crystals of different sizes and shapes depending on their position in the cloud, are located in the troposphere at altitudes above $8 \mathrm{~km}$. As most of the water-vapor is located below this altitude, Gao and al. ${ }^{2}$ (1993) showed, with AVIRIS hyperspectral instrument's data, that the exploitation of strong water-vapor absorption bands, like $1.38 \mu \mathrm{m}$ or $1.88 \mu \mathrm{m}$, allowed cirrus detection. The $1.38 \mu \mathrm{m}$ band was then integrated into various sensors: MODIS (Terra (1999) and Aqua (2002)), then VIIRS (Suomi-NPP, 2011), OLI (Landsat-8, 2013) and MSI (Sentinel-2, 2015). However, this detection method has limitations: dry atmosphere, high altitude or strong albedo surface and high water clouds ${ }^{3}$. Gao and al. proposed then an empirical method to correct thin cirrus, based on the utilization of the $1.38 \mu \mathrm{m}$ band. The method was initially limited to the [0.4-1.0 $\mu \mathrm{m}]$ region $^{4}$, then, extended to the SWIR for Landsat-8 ${ }^{5}$, in 2017.

Gao and al. method (1998) is today used in several processing chains, and has shown qualitatively its effectiveness in removing cirrus effects. In this paper, a quantitative validation of 2017'method is proposed.

The next section of this paper describes Gao and al. method, as well as the method and data used for validation. The 3rd part reports the results and finally, the method is discussed in the $4^{\text {th }}$ section. 


\section{MATERIEL AND METHOD}

\subsection{Cirrus detection}

Gao and $\mathrm{al}^{2}$ shows that the $1.38 \mu \mathrm{m}$ saturated water vapor absorption band allowed cirrus detection. Indeed in this band, the only signal reaching the sensor comes from the scattering in the upper cirrus cloud, all the signal coming from below being totally absorbed by the water vapor. This led Richter and al. ${ }^{6}$ to propose a cirrus classification. This classification is based on thresholds in the apparent reflectance of the cirrus band $(1.38 \mu \mathrm{m})$ as shown below:

- $\quad$ Clear sky (Ci0)

$: \rho^{*}(1.38 \mu \mathrm{m}) \in[0-0.01[$

- $\quad$ Semi-transparent thin cirrus (Ci1)

$: \rho^{*}(1.38 \mu m) \in[0.01-0.015[$

- Semi-transparent medium cirrus $(\mathrm{Ci} 2) \quad: \rho^{*}(1.38 \mu m) \in[0.015-0.025[$

- Semi-transparent thick cirrus (Ci3) $\quad: \rho^{*}(1.38 \mu \mathrm{m}) \in[0.025-0.04[$

- Optically thick thin cirrus (Ci4) $\quad: \rho^{*}(1.38 \mu m) \in[0.04-0.05[$

- Optically thick medium (Ci5) $\quad: \rho^{*}(1.38 \mu \mathrm{m}) \geq 0.05$

The $\rho^{*}(1.38 \mu \mathrm{m})=0.04$ separates semi-transparent cirrus from optically thick cirrus. These thresholds are used in the following for the analysis of the results.

\subsection{Cirrus correction: Gao and al. method}

\subsubsection{Empirical model}

Gao and al. $^{3}$ (1998) proposed a first empirical method for the correction of thin cirrus. This method is limited to the [0.4-1.0 $\mu \mathrm{m}]$ region where cirrus optical properties can be assumed spectrally flat. Beyond $1.0 \mu \mathrm{m}$, absorption effects modify the spectral signature.

The Earth-atmosphere system is modeled by 2 plan and parallel layers: a virtual surface (including the surface and all the atmosphere - water-vapor, water clouds, aerosols) and a layer of cirrus clouds.

Using the classic formula of $6 \mathrm{~S}^{7}$ and applying it to the cirrus layer, the measured apparent reflectance is written:

$$
\rho_{\text {measured }}^{*}(\lambda)=\rho_{c}^{*}(\lambda)+T_{c} \rho^{*}(\lambda) \cdot \frac{1}{1-S_{c} \rho^{*}(\lambda)},
$$

with $\rho^{*}{ }_{\mathrm{c}}$ the apparent reflectance of the cirrus cloud, $\mathrm{T}_{\mathrm{C}}$ the total transmission (diffuse + direct, up and down) through the cirrus, $\rho^{*}$ the apparent reflectance of the virtual, and $S_{c}$ the spherical albedo of the cirrus. From this equation some simplification are made : the coupling term is simplified, assuming $S_{c}$ is weak $(<0.2)$ and, total absorption being negligible for thin cirrus, the transmission is put equal to unity and, the apparent reflectance of the cirrus in the domain 0.4-1.0 $\mu \mathrm{m}$ is proportional to the signal at $1.38 \mu \mathrm{m}$ :

$$
\rho_{c}^{*}(\lambda)=\frac{\rho_{c}^{*}(1.38 \mu m)}{K_{a}},(0.4<\lambda<1.0 \mu \mathrm{m})
$$

The $\mathrm{K}_{\mathrm{a}}$ coefficient accounting for the residual water vapor absorption in the 1.38 band. The reflectance corrected from the presence of thin cirrus then expresses as:

$$
\rho^{*}(\lambda)=\rho_{\text {measured }}^{*}(\lambda)-\frac{\rho_{C}^{*}(1.38 \mu \mathrm{m})}{K_{a}},(0.4<\lambda<1.0 \mu \mathrm{m})
$$

In 2017 Gao and $\mathrm{Li}^{5}$ extended the method to the SWIR, for Landsat-8 (11 bands, from 0.4 to $12.5 \mu \mathrm{m}$ ), by modifying the formula 3 considering not just one coefficient $K_{a}$, but a $K_{a}$ depending on the wavelength:

2.2.2 Estimation of $K_{a}$ coefficient

$$
\rho^{*}(\lambda)=\rho_{\text {measured }}^{*}(\lambda)-\frac{\rho_{c}^{*}(1.38 \mu \mathrm{m})}{K_{a}(\lambda)}
$$


The coefficients $K_{a}(\lambda)$ are estimated by a scatterplot between the $1.38 \mu \mathrm{m}$ band and the band $\lambda$ (Fig.1). These coefficients depend on the scene, the cirrus, and on solar and view angles. The linear regression slope is obtained with the Gao and al. method $^{8}$, which consist in, for each bins in the cirrus apparent reflectance, taking the closest points to the minimum value of the apparent reflectance in the band $\lambda$. They also suggest a piecewise-linear line with $x$ slopes. But as the number of slopes and the threshold on $\rho^{*}(1.38 \mu \mathrm{m})$ depend on the image and the band, it is difficult to set it manually.
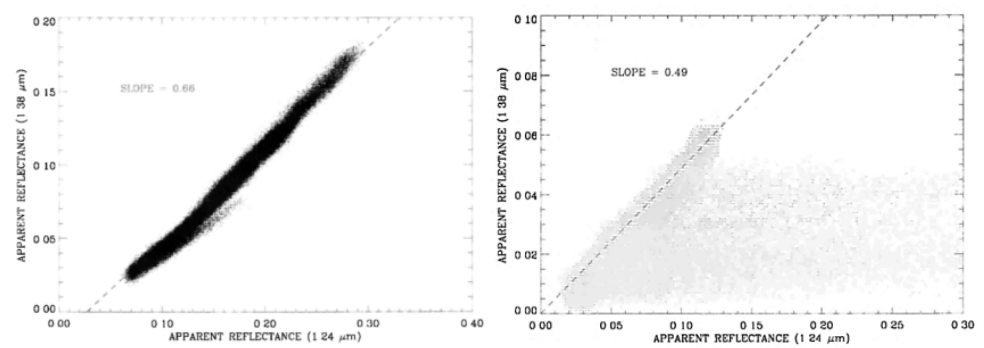

Figure 1: Example of a linear correlation between the 1.24 and $1.38 \mu \mathrm{m}$ bands for an AVIRIS image (Gao et al., 1998). On the left, the graph corresponds to a homogeneous surface image containing only cirrus type clouds. On the right, the pixels around the dotted line are the fine cirrus pixels. The points to the right of the line correspond to the cumulus. The same type of graph is obtained when correlating any $\lambda$ and $1.38 \mu \mathrm{m}$ bands.

\subsection{Data and validation method}

To validate the method, couples of Sentinel-2 images were used. Sentinel-2 is a series of 2 Earth observation satellites (S2A (2015) and S2B (2017)) from ESA (European Space Agency) developed for the Copernicus program. These satellites are equipped with a MSI multispectral imager using the pushbroom technique and having 13 bands from visible to SWIR with spatial resolution of 10, 20 and 60 meters. They are taken in Level-1C product (Top of Atmosphere radiance).

Thanks to the instrument high revisit, two acquisitions, one under clear sky conditions and the second with cirrus clouds, can be found in a short period time (less than 5 days) so that the ground scene can be assumed invariant and the solar angles almost identical. Then, on each pair of images, areas of interest were carefully selected. Once the cirrus image is corrected by the method previously described, it is compared with the corresponding clear sky image. For this, on each selected area the RMS difference is calculated between the corrected image and the clear sky reference, for each band, and according to the class of cirrus. Each downloaded Sentinel-2 granule has been cut to reduce computational time. 5 couples of images fulfilling the conditions were found: 2 couples of water images and 3 containing mixed surfaces of sea and continental surface (Table 1).

Table 1: Characteristics of selected sentinel-2 images

\begin{tabular}{|c|c|c|c|c|c|c|c|c|c|}
\hline S2 tile & Region & $\begin{array}{l}\text { Studied } \\
\text { area }\end{array}$ & Sky & Satellite & Date + UTC time & $\begin{array}{c}\text { Solar } \\
\text { zenithal } \\
\text { angle }\end{array}$ & $\begin{array}{c}\text { Solar } \\
\text { azimuthal } \\
\text { angle }\end{array}$ & $\begin{array}{c}\text { Zenithal } \\
\text { angle of } \\
\text { view }\end{array}$ & $\begin{array}{l}\text { Azimuthal } \\
\text { angle of } \\
\text { view }\end{array}$ \\
\hline \multirow{2}{*}{ T17TLL } & \multirow{2}{*}{$\begin{array}{l}\text { Huron Lake } \\
\text { (Canada) }\end{array}$} & \multirow{2}{*}{ Water } & Clear sky & S2B & $08 / 23 / 2018-16$ h18 & $36.8^{\circ}$ & $153.5^{\circ}$ & $8.3^{\circ}$ & $105^{\circ}$ \\
\hline & & & Cirrus & $\mathrm{S} 2 \mathrm{~B}$ & $08 / 26 / 2018-161228$ & $37.0^{\circ}$ & $158.2^{\circ}$ & $6.4^{\circ}$ & $284.5^{\circ}$ \\
\hline \multirow{2}{*}{ T37TOG } & \multirow{2}{*}{ Black Sea } & \multirow{2}{*}{ Water } & Clear sky & S2B & $10 / 28 / 2018-8 \mathrm{~h} 20$ & $56.1^{\circ}$ & $166.4^{\circ}$ & $6.0^{\circ}$ & $105.2^{\circ}$ \\
\hline & & & Cirrus & S2B & $10 / 31 / 2018-8 \mathrm{~h} 30$ & $56.7^{\circ}$ & $169.5^{\circ}$ & $8.9^{\circ}$ & $286.0^{\circ}$ \\
\hline \multirow{2}{*}{ T31TFJ } & \multirow{2}{*}{$\begin{array}{l}\text { La Crau } \\
\text { (France) }\end{array}$} & \multirow{2}{*}{$\begin{array}{c}\text { Water } \\
\text { La Crau plain }\end{array}$} & Clear sky & SLB & $07 / 29 / 2018-10130$ & $28.8^{\circ}$ & $145.0^{\circ}$ & $7.2^{\circ}$ & $106.5^{\circ}$ \\
\hline & & & Cirrus & S2A & $08 / 03 / 2018-10 \mathrm{~h} 30$ & $29.9^{\circ}$ & $146.2^{\circ}$ & $7.1^{\circ}$ & $105.5^{\circ}$ \\
\hline \multirow{2}{*}{ T30TXQ } & \multirow{2}{*}{ Landes (France) } & \multirow[b]{2}{*}{ Pine forest } & Clear sky & S2A & $09 / 11 / 2018-10 \mathrm{~h} 56$ & $41.8^{\circ}$ & $160.3^{\circ}$ & $2.9^{\circ}$ & $196.6^{\circ}$ \\
\hline & & & Cirus & SLB & $09 / 16 / 2018-10156$ & $43.5^{\circ}$ & $161.6^{\circ}$ & $2.8^{\circ}$ & $193.6^{\circ}$ \\
\hline \multirow{2}{*}{ T29TNF } & \multirow{2}{*}{ Portugal } & \multirow[b]{2}{*}{ Forest } & Clear sky & S2A & $10 / 25 / 2017-11 \mathrm{~h} 33$ & $54^{\circ}$ & $168.7^{\circ}$ & $9.0^{\circ}$ & $287.3^{\circ}$ \\
\hline & & & Cirrus & S2A & $10 / 22 / 2017-11 \mathrm{~h} 21$ & $53.4^{\circ}$ & $165.3^{\circ}$ & $6.0^{\circ}$ & $103.8^{\circ}$ \\
\hline
\end{tabular}




\section{RESULTS}

\subsection{Cirrus detection}

The ATCOR cirrus classification is applied on the different selected images. Fig.2 shows an illustration of this classification for the La Crau site. The different thicknesses of cirrus appear well distinct and contrails are also detected.

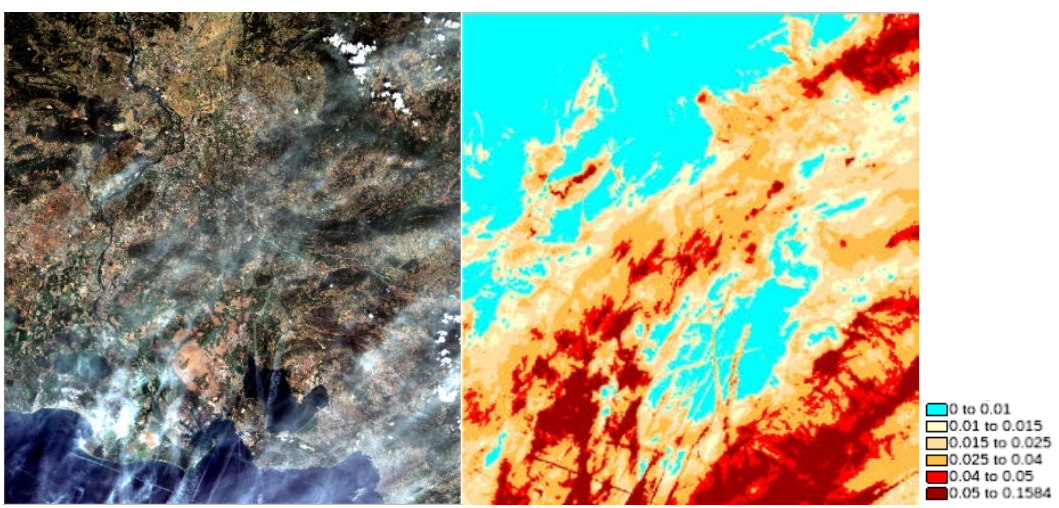

Figure 2: Left: RGB S2 image of La Crau region. Right: Classification of the cirrus with threshold in the apparent reflectance at $1.38 \mu \mathrm{m}$

\subsection{Cirrus correction}

Fig.4(a). shows how the presence of cirrus affects the apparent reflectance for pixels of water, inducing a positive offset, whatever the wavelength. The curve after application of Gao and Li method fit very well the clear sky curve, showing an offset corrected for this pixel. Then, for each studied area, the RMS difference is calculated in each band and each class of cirrus according to the ATCOR classification. In the table 2 below, an average value per band group of the RMS values of each band is presented. The bands were grouped into 3 groups: those before the red-edge, those of the NIR and those of the SWIR.

Table 2: RMS error for each studied area, and for each group of band and class of cirrus. In red region with high RMS values.

\begin{tabular}{|c|c|c|c|c|c|c|c|c|c|c|c|c|c|c|c|c|c|c|}
\hline \multirow{2}{*}{ Area } & \multirow{2}{*}{\multicolumn{6}{|c|}{$[0.4-0.7 \mu \mathrm{m}]$}} & \multicolumn{6}{|c|}{$[0.7-0.86 \mu \mathrm{m}]$} & \multicolumn{6}{|c|}{ SWIR } \\
\hline & & & & & & & C10 & CII & CI2 & $\mathrm{C13}$ & $\mathrm{C} 14$ & $\mathrm{C15}$ & C10 & CII & CI2 & $\mathrm{C} 13$ & $\mathrm{C} 14$ & $\mathrm{C15}$ \\
\hline Huron lake & 0.005 & 0.006 & 0.008 & 0.008 & 0.008 & 0.008 & 0.004 & 0.008 & 0.008 & 0.009 & 0.012 & 0.013 & 0.004 & 0.007 & 0.008 & 0.007 & 0.009 & 0.009 \\
\hline Black Sea & 0.008 & 0.030 & 0.038 & 0.026 & 0.037 & 0.012 & 0.009 & 0.035 & 0.045 & 0.029 & 0.041 & 0.011 & 0.005 & 0.015 & 0.018 & 0.013 & 0.018 & 0.009 \\
\hline $\begin{array}{c}\text { Portugal - } \\
\text { water }\end{array}$ & 0.005 & 0.006 & 0.006 & 0.007 & 0.008 & 0.01 & 0.002 & 0.004 & 0.005 & 0.008 & 0.01 & 0.012 & 0.002 & 0.003 & 0.003 & 0.004 & 0.006 & 0.009 \\
\hline $\begin{array}{l}\text { Landes - } \\
\text { water }\end{array}$ & 0.002 & 0.003 & 0.005 & 0.007 & 0.009 & 0.01 & 0.002 & 0.002 & 0.004 & 0.006 & 0.007 & 0.01 & 0.002 & 0.002 & 0.004 & 0.005 & 0.006 & 0.007 \\
\hline Crau-water & 0.006 & 0.005 & 0.007 & 0.01 & 0.015 & 0.021 & 0.006 & 0.008 & 0.016 & 0.027 & 0.039 & 0.058 & 0.004 & 0.005 & 0.007 & 0.011 & 0.017 & 0.026 \\
\hline Mean RMS & 0.0058 & 0.011 & 0.0097 & 0.0079 & 0.0116 & 0.0178 & 0.0053 & 0.013 & 0.011 & 0.013 & 0.026 & 0.047 & 0.004 & 0.006 & 0.006 & 0.0064 & 0.012 & 0.021 \\
\hline $\begin{array}{c}\text { Portugal - } \\
\text { forest }\end{array}$ & 0.004 & 0.004 & 0.004 & 0.004 & $\mathrm{x}$ & $\mathrm{x}$ & 0.014 & 0.015 & 0.011 & 0.008 & $\mathrm{x}$ & $\mathrm{x}$ & 0.006 & 0.007 & 0.006 & 0.004 & $\mathrm{x}$ & $\mathrm{x}$ \\
\hline $\begin{array}{c}\text { Landes - } \\
\text { forest }\end{array}$ & 0.005 & 0.006 & 0.007 & $\mathrm{x}$ & $\mathrm{x}$ & $\mathrm{x}$ & 0.021 & 0.017 & 0.017 & $\mathrm{x}$ & $\mathrm{x}$ & $\mathrm{X}$ & 0.009 & 0.008 & 0.09 & $\mathrm{x}$ & $\mathrm{x}$ & $\mathrm{x}$ \\
\hline Mean RMS & 0.0042 & 0.0058 & 0.0068 & 0.004 & $\mathrm{x}$ & $\mathrm{x}$ & 0.0158 & 0.0168 & 0.0165 & 0.008 & $\mathrm{X}$ & $\mathrm{X}$ & 0.0068 & 0.0079 & 0.085 & 0.004 & $\mathrm{X}$ & $\mathrm{X}$ \\
\hline Crau - plain & 0.006 & 0.008 & 0.014 & 0.031 & 0.038 & 0.037 & 0.012 & 0.015 & 0.030 & 0.066 & $\overline{0.080}$ & 0.077 & 0.022 & 0.028 & 0.047 & 0.054 & 0.103 & 0.087 \\
\hline
\end{tabular}

On water, results show that the reflectance error is generally found to be less than 0.02 , for all bands and even for the thickest cirrus. However, for images of the Black Sea and La Crau, larger values are observed. The common feature between these two images is the presence of contrails. In the Black Sea case, these values are due to the $K_{a}$ coefficients. Indeed, Fig.3 shows that the pixels used for the calculation of $K_{a}(\lambda)$ correspond to the contrails pixels (in red in the Fig). The green and blue groups correspond to the cirrus and exhibit a different slope. Only the contrails are well corrected while cirrus were under-corrected. On the image of La Crau region, the pixels used for calculating the $\mathrm{K}_{\mathrm{a}}$ coefficients 
correspond to cirrus above the water, but here the contrails pixels "sign" and do not just cause any offset (Fig.4.(b)) therefore, after correction, the spectrum found is not consistent with water signature.

Once the correction on the water analyzed, the study was done on continental surfaces. Forests are dark and therefore present an apparent reflectance in clear sky which remains weak. This case is similar to that of water, except above the red-edge. By the bye, it's in this region where the RMS values are a little larger and raise up to 0.021. On the rest of the spectrum, RMS values remain less than 0.01 .

The La Crau plain, on the other hand, has higher apparent reflectance values in clear sky, especially in the red-edge and SWIR regions where the values are higher than 0.2. It is in these same 2 regions that high RMS values (>0.03) are found after correction. In the visible, thicker cirrus are also poorly corrected.

Globally, Gao's method delivers consistent results and finds apparent reflectance values within 0.02 . The thicker the cirrus is, the less accurate the correction will be. Similarly, larger uncertainties are obtained with highly reflective surfaces. $K_{a}(\lambda)$ estimation seems to play a major role in the accuracy of the correction.

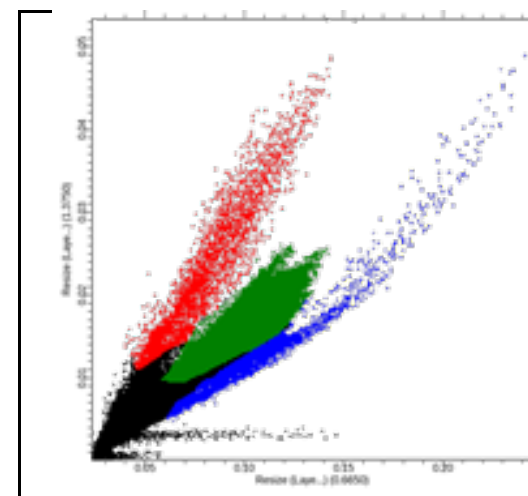

(a)

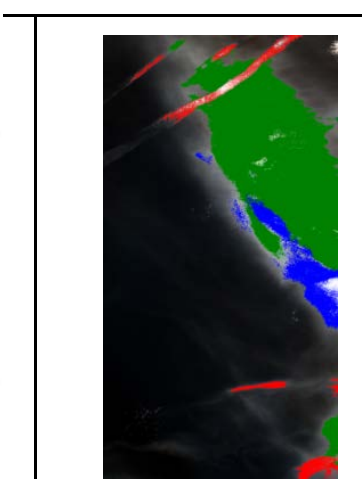

(b)

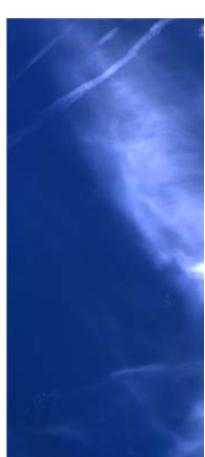

( c )

Figure 3: (a) Scatterplot of the $1.38 \mu \mathrm{m}$ band with the $0.66 \mu \mathrm{m}$ band with the different groups in color. (b) The Sentinel-2 image in the $1.38 \mu \mathrm{m}$ band with the color pixels of the associated groups. (c) RGB image
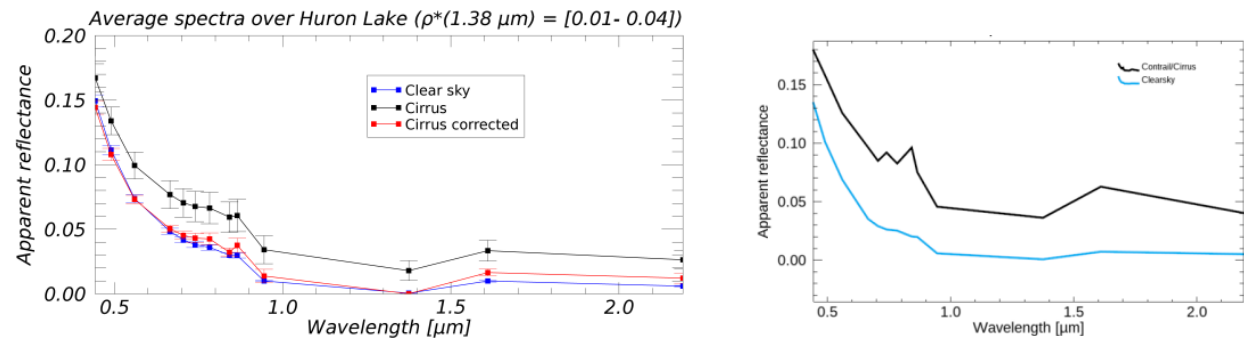

Figure 4: (a): Average spectral profile of pixels of water (Huron Lake) in the cirrus image (black), in the clear-sky image (blue), and after correction (red). (b): Spectral profile of a pixel of contrails (black) and a pixel of clear sky (blue) over the water (La Crau).

\section{DISCUSSION}

\subsection{Estimation of the Ka coefficient}


The formula 4, as the Black Sea example, shows that the accuracy of the correction depends largely on the quality of $K_{a}(\lambda)$ estimation. Depending on the situation, the estimation is more or less simple. When scatterplots are usually in the shape of the left Fig. 1 for water images with an easy slope to estimate, the presence of contrails modifies the scatterplot and "falsifies" the correction (Fig.3).

A test on this image has been done. 3 slopes were calculated, corresponding to the 3 groups of Fig.3, and the pixels of each group were corrected with the corresponding $K_{a}$. Fig.5 shows the results of RMS with the use of a single $K_{a}$ for all the pixels (a) and with the use of coefficients depending on the pixel (here 3 different $K_{a}(\lambda)$ ) (b). The correction is improved for thin cirrus and we are left with similar cases to other images. For thicker cirrus, the RMS is higher but it is possible that they were not well selected and thus corrected with the wrong value of $K_{a}(\lambda)$. Without contrails, the image would have been well corrected.

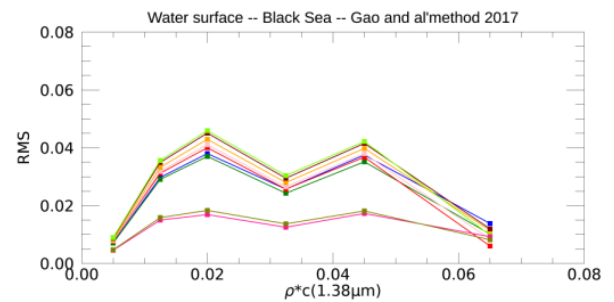

(a)
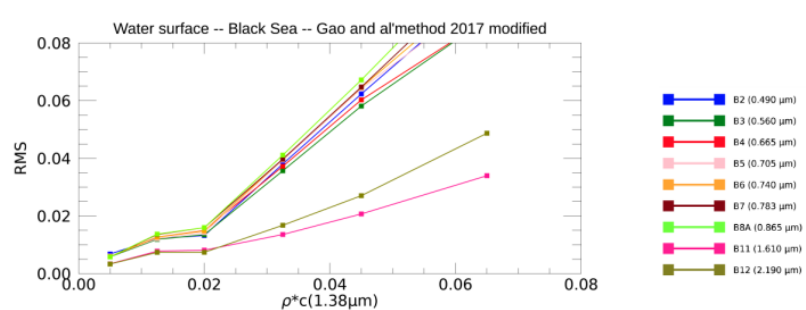

(b)

Figure 5: RMSE function of the thickness of the cirrus, for each band. (a): when applyingGao and al 2017 method. (b): Gao and al. 2017 method with different coefficients $K_{a}$.

Furthermore, for more complex images with several types of surfaces, the scatter plot may be different depending on the bands and surfaces present, and the estimation of $K_{a}(\lambda)$ becomes more complex. Let's take the example of scatterplot between the bands 1.38 and $0.78 \mu \mathrm{m}$ of the S2 La Crau image below (Fig.6.(a)). If we calculate the slope using all the points of the left envelope, the slope will have a value of 0.395 . Moreover, if we do not exclude the points circled in Fig.6.(a), the calculation of the slope also differs with a value of 0.369. If now we decide to set a threshold manually, at $\rho^{*}(1.38 \mu \mathrm{m})=0.05$ where the cirrus are starting to get very thick, and calculate the slope only up to this value, we get a slope of 0.835. Fig.6.(b) shows the RMS as a function of the wavelength, calculated for every cirrus on La Crau plain, with these 3 different estimation of the coefficients $K_{a}(\lambda)$. This show that a different value of $K_{a}(\lambda)$ can completely change the accuracy. For this image, and others, the result is better when the threshold is fixed (orange curve), particularly in the NIR bands, where the RMS is less than 0.02 instead of 0.05 .

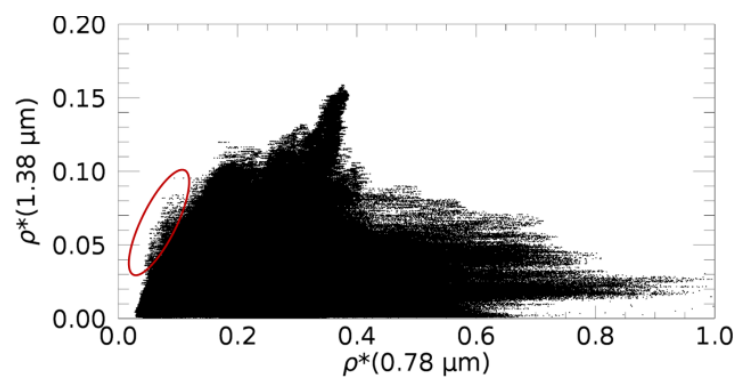

(a)

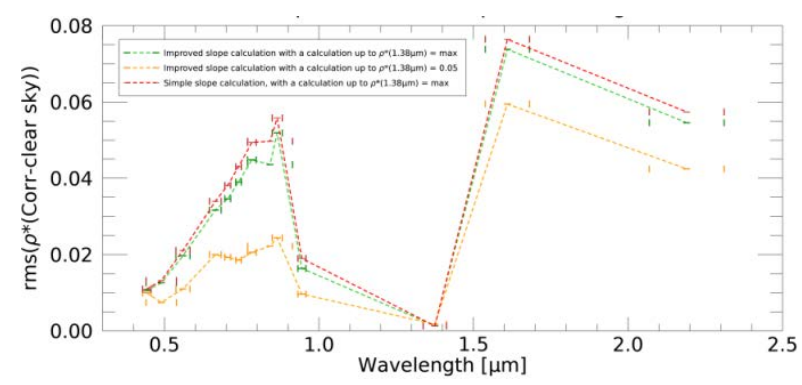

(b)

Figure 6: (a): Scatterplot between the apparent reflectance at $1.38 \mu \mathrm{m}$ and at $0.78 \mu \mathrm{m}$ for the S2 image over the La Crau region. (b): RMS as a function of the wavelength, calculated for all cirrus, for 3 different corrections, for the La Crau plain. In red, with a $K_{a}(\lambda)$ calculated with all the pixel of the left envelope of the scatterplot; in green, with a $K_{a}(\lambda)$ calculated after filtering point; in orange, with a $\mathrm{K}_{\mathrm{a}}(\lambda)$ calculated only up to $\rho^{*}(1.38 \mu \mathrm{m})=0.05$. 


\subsection{Limitation}

The image above La Crau site raised many questions. Some pixels of La Crau region were over-corrected. Looking closer, it was found that in some cases, in the 0.7-0.9 $\mu \mathrm{m}$ region, the signal of the cirrus image is lower than that of the clear sky image. We found that this case correspond, most of the time, to the cirrus shadow as we can see in the figure 7 were patterns are found.
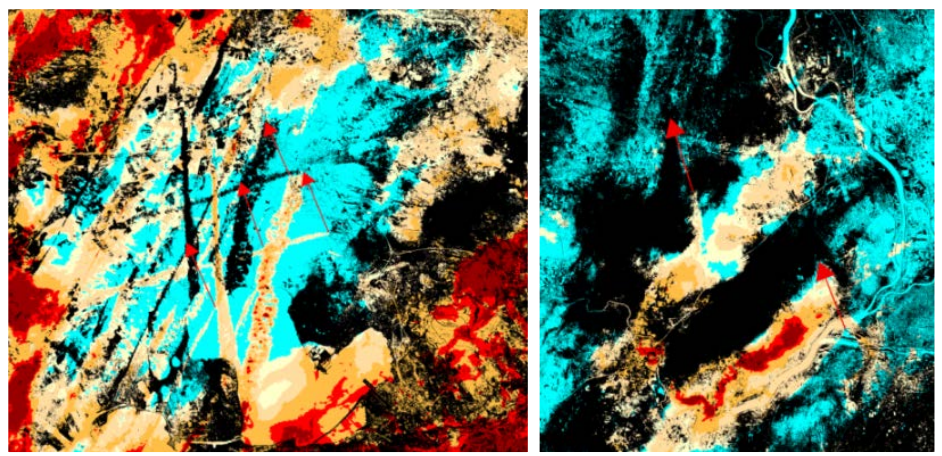

Figure 7: 2 zooms on the image of the Crau, showing the different classes of cirrus and, in black, the pixels where the apparent reflectance in the band 8A in the presence of cirrus is lower than that in clear sky. Red arrows show that it seems to match the shadows of the cirrus.

Gao and Li model only corrects the rising path. However, the downward path has a significant impact on the signal, especially for surfaces of strong apparent reflectance. These observations led to simulations, with COMANCHE ${ }^{9}$, to understand how the presence of cirrus in the downward path modifies the apparent reflectance and then propose an improvement of the correction.

14 cirrus are simulated in the conditions of the Sentinel-2 image of La Crau, and 3 possible situations are studied (Fig.8): An homogeneous cirrus covering the entire scene, an homogeneous cirrus only in the downward path and an homogeneous cirrus only on the upwelling path.

We focus here on the $0.8 \mu \mathrm{m}$ wavelength, where we noticed the problems. The analysis of the difference between the signal in presence of cirrus and the signal in clear sky show different results according to the situation. When we see clear sky on the image, the received signal may be lower than the actual signal if a cirrus is present on the downward path, especially if the surface has a strong reflectance and if the cirrus on the down path is optically thick. This can cause errors up to 0.06 on the signal before the correction. When the cirrus is only on the rising path, there is mainly an increase on the apparent reflectance. But when the cirrus is thin and the surface has a strong reflectance there is an inversion, and the signal is reduced. The last case is a combination of the 2 other. It would be necessary to be able to identify these different situations in the image in order to apply the appropriate correction. 


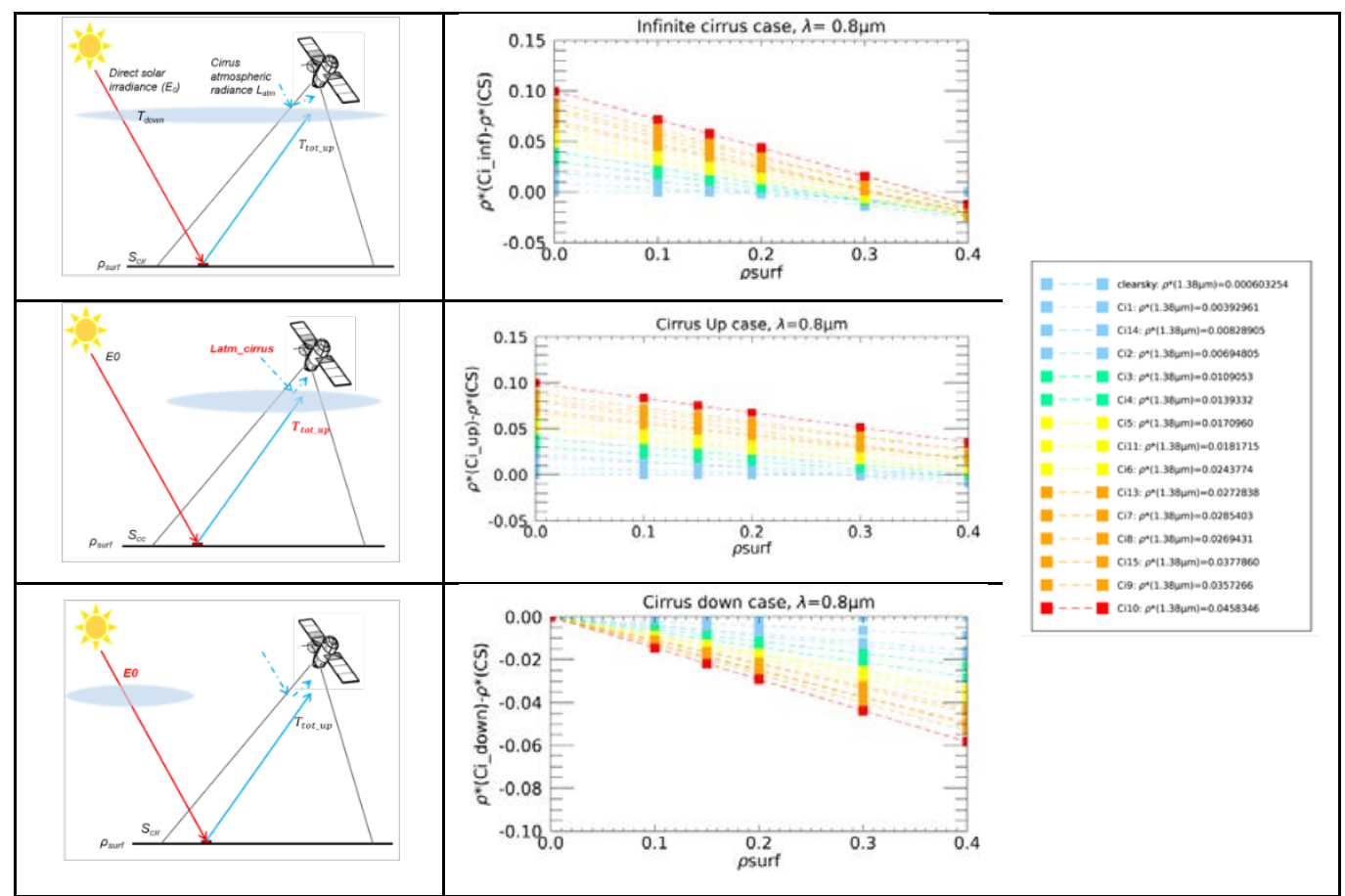

Figure 8: Left: Different case of simulations. Right: Difference between the apparent reflectance in presence of cirrus and in clear sky as a function of the surface reflectance, for the case of simulation corresponding, at $\lambda=0.8 \mu \mathrm{m}$

\section{CONCLUSION}

The method of thin cirrus correction of Gao and Li extended to the SWIR applied on Sentinel-2 data globally shows good results. On dark surfaces such as water or forest, the apparent reflectance in clear sky is found to 0.02. But on more reflective surfaces, such as La Crau plain, results are worse, especially in the NIR bands, where the RMS values are greater than 0.03 .

The model is limited by several things. First, $K_{a}(\lambda)$ estimation plays a fundamental role in the accuracy of the correction. The latter can sometimes be distorted by the presence of contrails. Then, the model only corrects the rising path here. However, the analysis of the images show that for some pixels, the apparent reflectance in the presence of cirrus is lower than that in clear sky, causing an over-correction when applying the formula. This weaker reflectance appears to be caused by the presence of a cirrus on the descending path (sun-ground). This is supported by the various simulations. Knowing how to separate the pixels in the cirrus 'shadow from the others, with the help of the single cirrus image, would make it possible to apply two different corrections and to improve the accuracy of the correction.

\section{REFERENCES}

[1] Stubenrauch, C.J., Chédin, A., Rädel, G., Scott, N.A. and Serrar, S., “Cloud Properties and Their Seasonal and Diurnal Variability from TOVS Path-B”, American Meteorological Society, 5531-5553 (2006)

[2] Gao, B.C. and Goetz, A.F.H., "Cirrus cloud detection from airborne imaging spectrometer data using the 1.38 $\mu \mathrm{m}$ water vapor band”, Geophysical Research Letter 20, 301-304 (1993)

[3] Ben-Dor, E., “A precaution regarding cirrus cloud detection from airborne imaging spectrometer data using the 1.38/am Water vapor band”, Remote Sensing Environment 50, 346-350 (1994) 
[4] Gao, B.C., Kaufman, Y.J., Han, W. and Wiscombe, W.J., "Correction of thin cirrus path radiance in the 0.4-1.0 $\mu \mathrm{m}$ spectral region using the sensitive $1.38 \mu \mathrm{m}$ cirrus detecting channel”, Journal of Geophysical Research 103, 32.169-32.176 (1998)

[5] Gao, B.C and Li, R.R., "Removal of thin cirrus scattering effects in Landsat 8 OLI images using the cirrus detecting channel”, Remote Sensing 9, 834 (2017)

[6] Richter, R. and Schläpfer, D. [Atmospheric and Topographic Correction (ATCOR Theoretical Background Document)], DLR IB 564-03/19, Wessling, Germany (2019)

[7] Vermote, E., Tanré, D., Deuzé, J.L. and Herman, M., [Second Simulation of a Satellite Signal in the Solar Spectrum - Vector (6SV)] (2006)

[8] Gao, B.-C., Yang, P., Han, W., Li, R.-R. and Wiscombe, W.J., “An algorithm using visible and 1.38-micro, channels to retrieve cirrus cloud reflectances from aircraft and satellite data.” IEEE Transactions on Geoscience and Remote Sensing, 1659-1668 (2002)

[9] Poutier, L., Miesch, C., Lenot, X., Achard, V. and Boucher, Y., “COMANCHE and COCHISE: two reciprocal atmospheric codes for hyperspectral remote sensing” (2002) 